\title{
Efficacy and Safety of Viusid and Asbrip in Hospitalized Patients with Mild and Moderate COVID-19: A Randomized Controlled Trial
}

\author{
Petar Petrov', Aleksandar Mihaylov², Maria Shopova1, Maria Boncheva1, David Marquez ${ }^{3}$ \\ ${ }^{1}$ Covid Department, MBAL “Sveti Mina”, Plovdiv, Bulgaria \\ ${ }^{2}$ Covid Department, MTB-Plovdiv, Plovdiv, Bulgaria \\ ${ }^{3}$ Scientific Department, Catalysis Laboratories, Madrid, Spain \\ Email: prof.petrov@abv.bg, aleksandar.mihaylov@gmail.com,m.shopova04@abv.bg, maria.boncheva@mail.ru, \\ david@catalysis.es
}

How to cite this paper: Petrov, P., Mihaylov, A., Shopova, M., Boncheva, M. and Marquez, D. (2021) Efficacy and Safety of Viusid and Asbrip in Hospitalized Patients with Mild and Moderate COVID-19: A Randomized Controlled Trial. Advances in Infectious Diseases, 11, 171-184.

https://doi.org/10.4236/aid.2021.112017

Received: March 25, 2021

Accepted: May 16, 2021

Published: May 19, 2021

Copyright $\odot 2021$ by author(s) and Scientific Research Publishing Inc. This work is licensed under the Creative Commons Attribution International License (CC BY 4.0).

http://creativecommons.org/licenses/by/4.0/

\begin{abstract}
Background: The aim of this study was to test Viusid and Asbrip as adjuvants to the standard treatment of patients with COVID-19 disease which could favor the recovery of the patients and reduce the hospitalization days. Design: This is a randomized, open-label, controlled trial to determine the efficacy and safety of Viusid and Asbrip in hospitalized patients with mild to moderate symptoms of COVID-19. Material and Methods. A total of 60 patients with proven COVID-19 disease by PCR test were randomized in a 2:1 ratio. In the active group 40 patients received oral doses of Viusid (30 ml TID) and Asbrip (10 ml TID) plus standard treatment. The control group consisted of 20 patients receiving only standard treatment. The trial began with hospitalization, followed by home treatment for a total of 21 days. Four symptoms were followed: fever, dyspnea, cough and fatigue, assessed by score $0-3: 0=$ well, $1=$ mild, $2=$ moderate, $3=$ severe, with total sum, Composite Symptom Score (CSS) from 0 to 12. Results: The 21 days diagram of CSS shows statistically better results of Viusid-Asbrip group vs. Control group from day 4 to day 21 . Time to semi-recovery in days, assessed by $50 \%$ of CSS, is better in Viusid-Asbrip group vs. Control group ( $6.07 \pm 2.77$ vs. $8.35 \pm 2.94, \mathrm{p}=0.02)$. Time to recovery in days respectively is $(14.05 \pm 4.15$ vs. $19.25 \pm 2.12, \mathrm{p}=$ $0.0001)$. And Hospitalization days are $(9.05 \pm 2.58$ vs. $12.75 \pm 4.44, \mathrm{p}=$ 0.0003). Conclusion: This trial shows that adding of Viusid \& Asbrip to the treatment of COVID-19 can contribute to faster recovery of the patients, decreasing of the hospital stay and milder course of the disease.
\end{abstract}

\section{Keywords}

Immunomodulator, COVID-19, Hospitalization, Recovery, Antioxidant 


\section{Introduction}

Beginning of December 2019, a new infectious respiratory disease outbroke in Wuhan City, Hubei Province in China. On 31 December 2019, the World Health Organization (WHO)-China Country Office, was informed of cases of pneumonia of unknown etiology (unknown cause) detected in Wuhan City [1]. The responsible pathogen is a novel coronavirus, named severe acute respiratory syndrome coronavirus 2 . WHO officially named this infectious disease coronavirus disease 2019 (COVID-19) on 12 February 2020 [2]. Later, the International Committee on Taxonomy of Viruses (ICTV) officially designated the virus as SARS-CoV-2 based on phylogeny, taxonomy and established practice [3]. Currently, people all over the world have been affected by COVID-19, which is the fifth pandemic after the 1918 flu pandemic [4]. Till the end of Feb. 2021, the infected people in the world reached 115,000,000 and 2,500,000 deaths were registered.

The virus SARS-CoV-2 passes through the mucous membranes of the nose and larynx, enters the lungs through the airways and peripheral blood from the lungs, causes viremia [5] and then attacks many organs that express the angiotensin-converting enzyme-2 (ACE2) receptors, present in the lungs, heart, blood vessels, kidneys and gastrointestinal tract [6]. The SARS-CoV-2 recognizes human ACE2 more efficiently increasing the ability to transmit from person to person [7]. And ACE2 is a functional SARS-CoV receptor in vitro [8] and in vivo [9]. Thus, the SARS-CoV-2 spike protein was predicted to also have a strong binding affinity to human ACE2. It was demonstrated that overexpressing ACE2 from different species in HeLa cells allowed SARS-CoV-2 infection and replication, thereby directly showing that SARS-CoV-2 uses ACE2 as a cellular entry receptor [10]. ACE2 tissue distribution in the lung and other organs could explain the multi-organ dysfunction observed in patients [11]. In summary, the SARS-CoV-2 spike protein directly binds with the host cell surface ACE2 receptor facilitating virus entry and replication [6].

Due to the current epidemiological situation and the lack of a specific treatment protocol, different countries adhere to a different therapeutic approach. However, there appear some specific basic guidelines for the treatment. These include: antiviral, immunological, antibiotic, antithrombotic, booster therapy and vaccines. For example the US NIH guideline: "COVID-19 Treatment Guidelines", addresses two broad categories of therapies currently used to treat COVID-19: antiviral therapies and receiver modifiers, and therapies based on immunity [12].

Many drugs were investigated as antiviral therapy: Chloroquine or Hydroxychloroquine with or without Azithromycin; Lopinavir/Ritonavir and Other HIV Protease Inhibitors; Remdesivir; Ivermectin etc. But Remdesivir is the only FDA-approved drug for the treatment of COVID-19 [12]. In addition to antiviral drugs, adjuvant therapies are also used for the treatment of patients with COVID-19 for immune therapy and to prevent and treat the infection and its 
complications. An investigation found 38 Chinese drugs that are commonly used in the respiratory diseases and docked them against two drug targets, ACE2 receptor and viral main protease, might be of value for clinical trials during a 2019-nCov outbreak [13]. Other study shows five natural compounds: baicalin, scutellarin, hesperetin, nicotianamine and glycyrrhizin, that could have potential anti-2019-nCoV effects with capacity for binding ACE2, which may subsequently prevent the 2019-nCoV infection [14].

Glycyrrhizin or Glcsyrrhizinic acid (GA) is natural compound with antiviral, anti-inflammatory and immunomodulation properties. Different studies suggest that GA intervenes in direct inactivation of virus-associated kinase $P$ (SRC's family of kinases; SFK) and the reduction of the viral infectivity and replication [15] [16] [17] [18]. Furthermore, GA disrupt raft lipidic, that is so interesting as a new target for SARS-Cov-2 infection [19]. The anti-inflammatory and immunomodulation effect of GA is connected with regulation of macrophages, inhibiting pro-inflammatory cytokines and stimulating anti-inflammatory cytokines [20]. GA downregulates the secretion of IL- $1 \beta$, IL-3, IL-4, IL-5, IL-6, IL-12, IL-13, eotaxin, and TNF- $\alpha$ expression, and increases IFN- $\gamma$, important to control cytokine storms during viral diseases, especially for the management of patients with COVID-19 disease in the current SARS-2 coronavirus pandemic [21].

Viusid is natural product containing Glycyrrhizinic acid, malic, ascorbic and folic acids, glucosamine, arginine, glycine, calcium pantothenate, cyanocobalamine, zinc sulfate, pyridoxal. GA and the other important ingredients define Viusid's antiviral, anti-inflammatory and immunomodulatory properties. Moreover, Viusid has shown that help to increase production of interferon gamma [22]. Viusid showed its efficacy in many trials against hepatitis C virus [22], cirrhosis [23], influenza virus [24], HPV [25]. Also Viusid has proved very high anti-oxidant activity $(11,587.95 \mu \mathrm{mol} \mathrm{TE} / \mathrm{ml})$ measured in Brunswick Laboratories [26]. In conclusion Viusid is appropriate agent as addition to the treatment of the patients with mild and moderate COVID-19 disease. First report of application of Viusid clinical case of patient with atypical bilaterally viral pneumonia with RT-PCR proven coronavirus have been presented, with 16 days hospitalization and after two negative RT-PCR tests the patient was discharged in improved condition [27]. Clinical trial concluded use of Viusid was shown to be effective in the patients confirmed as having COVID-19, based on the scientific evidence of the benefits of each of its components. Given the safety of these treatments, they can be used in people of all ages within the established pharmacological dosing limits [28].

Asbrip is the second agent of the trial, natural compound based on eucalypt oil, malic acid, ascorbic acid and fumaric acid, cough syrup with faster broncholytic and expectorant action. Clinical trial assessed efficacy of the combination Asbrip + Viusid in the patients with chronic pharyngitis, with $95.7 \%$ efficacy and no side effects [29].

A clinical trial was completed in Ecuador with Asbrip and Viusid in mild and moderate pre-hospitalized COVID-19 patients. Preliminary results showed good 
recovery and shortened in-hospital stays with adjuvant protocol [30]. It seems that the combination Viusid + Asbrip can be applied as addition to the treatment of COVID-19 patients. Of course more trials have to confirm efficacy and safety of the products.

\section{Material and Methods}

Patients and study design: This is a two-arm, open-label, controlled, randomized, two-center clinical trial to determine the efficacy and safety of Viusid and Asbrip in hospitalized patients with mild to moderate symptoms of Covid-19 respiratory disease caused by SARS CoV-2 virus. The trial was performed in two hospitals in Plovdiv, Bulgaria, in the period Dec 2020-Feb 2021. The patients have proven coronavirus disease with a positive PCR test. A total of 60 patients were randomized in a 2:1 ratio in this study. In the active group 40 patients were assigned to receive Viusid and Asbrip, plus standard treatment. The control group consisted of 20 patients receiving only standard treatment. Treatment duration is 21 days and covers hospitalization and subsequent home treatment.

\section{Inclusion Criteria:}

1) Male or female adults aged $\geq 18$ years at the time of enrollment.

2) Subjects with mild-to-moderate symptoms of respiratory illness caused by coronavirus 2019 infection as defined below:

\section{Mild (uncomplicated) Illness:}

- Diagnosed with COVID-19 by a standardized RT-PCR assay and

- Mild symptoms, such as fever, rhinorrhea, mild cough, sore throat, malaise, headache, muscle pain, or malaise, but with no shortness of breath and

- No signs of a more serious lower airway disease and

- $\mathrm{RR}<20, \mathrm{HR}<90$, oxygen saturation (pulse oximetry) $>93 \%$ on room air Moderate Illness:

- Diagnosed with COVID-19 by a standardized RT-PCR assay and

- In addition to symptoms above, more significant lower respiratory symptoms, including shortness of breath (at rest or with exertion) or

- Signs of moderate pneumonia, including $R R \geq 20$ but $<30, H R \geq 90$ but less than 125, oxygen saturation (pulse oximetry) $>93 \%$ on room air and

- If available, lung infiltrates based on X-ray or CT scan $<50 \%$ present.

3) Clinically normal resting 12-lead ECG at screening visit or, if abnormal, considered not clinically significant by the Principal Investigator.

\section{Exclusion Criteria:}

1) Subjects showing signs of acute respiratory distress syndrome (ARDS) or respiratory failure necessitating mechanical ventilation at the time of screening.

2) History of severe chronic respiratory disease and requirement for long-term oxygen therapy.

3) Subjects showing signs of clinical jaundice at the time of screening.

4) History of moderate and severe liver disease (Child-Pugh score $>12$ ).

5) Subjects requiring Renal Replacement Therapy (RRT) at the time of screen- 
ing.

6) History of uncontrolled diabetes.

7) History of severe chronic kidney disease or requiring dialysis.

8) Any uncontrolled active systemic infection requiring admission to an intensive care unit (ICU); Note: Subjects infected with chronic hepatitis B virus or hepatitis $\mathrm{C}$ virus will be eligible for the study if they have no signs of hepatic decompensation.

9) Patients with malignant tumor, or other serious systemic diseases.

10) Patients who are participating in other clinical trials.

11) Patients who have a history of allergic reactions attributed to compounds of similar chemical or biologic composition to Viusid or Asbrip are not eligible.

Note: Empirical antibiotic treatment for secondary bacterial infections is allowed during the study.

Group treatments.

Viusid-Asbrip group: Each patient receives oral doses of Viusid, $30 \mathrm{ml}$ and Asbrip, $10 \mathrm{ml}$ every 8 hours, for 21 days. Standard care of the hospital was given to the patients, based on the clinical condition of each patient. After discharge, patients continue to take Viusid and Asbrip till day 21.

Control group: The patients in this group received only standard care consistent with the clinical condition of each patient, for 21 days.

The standard care of the hospital: Based on the next list, depending from the clinical condition of each patient:

1) antibiotic treatment (ceftriaxon, levofloxacin, cefoperazone);

2) symptomatic fever treatment (paracetamol, metamizole);

3) oxygen therapy in case of Sat. $<90 \%$;

4) dexamethazone in case of $\mathrm{T}>38^{\circ} \mathrm{C}$;

5) $0.9 \%$ sodium chloride infusion;

6) vitamins $C, D, B$ complex;

7) anticoagulant treatment (fraxiparin, dabigatran, apixaban);

8) statins-rouzvastatin.

\section{Outcome Measures}

\subsection{Primary Outcome Measures}

Clinical Improvement. Assessed by change in symptom scores. The symptom scores included: [everyday assessment, up to 21 days].

1) Fever Score (temperature in ${ }^{\circ} \mathrm{C}$ ) based on a scale 0 - 3: $0<36.7 ; 1=36.7$ $37.8 ; 2=37.8-38.9 ; 3>38.9$.

2) Cough Score on a scale $0-3: 0=$ no cough, $1=$ mild, $2=$ moderate, $3=$ severe.

3) Dyspnea Score based on a scale 0 - 3: $0=$ no dyspnea, $1=$ with moderate intensity exercise, 2 = with walking on flat surface, 3 = with getting dressed or daily activities.

4) Fatigue Score on a 0 - 3 scale: $0=$ no fatigue, $1=$ mild fatigue, $2=$ moderate 
fatigue, 3 = severe fatigue.

Composite symptom score (CSS): The sum of mentioned four symptom scores, assessed every day up to 21 days. It ranges from 0 to 12 .

Time to semi-recovery. Determined by the number of days required to reach a 50 percent reduction in CSS.

\subsection{Coprimary Outcome Measures}

Symptom resolution: The number of days required to reach average symptom score $<0.25$. It is for each one of four symptom category mentioned before, separately. We suppose that the value 0.25 must be defined by the average results of every four patients less than $[1,0,0,0]$, on each symptom.

Time to recovery. The number of days required to reach average CSS $<1$. The value 1 is defined by the average result of every four patients with symptom scores 0.25 .

\subsection{Secondary Outcome Measures}

Cumulative assessment of disease severity. Disease severity will be measured using a disease severity clinical event scale. Change from baseline in the patient's health status on an ordinal scale of 7 categories: [time frame: 21 days].

1) Death.

2) Hospitalized, with invasive mechanical ventilation or extracorporeal membrane oxygenation (ECMO).

3) Hospitalized, with non-invasive ventilation or high-flow oxygen devices.

4) Hospitalized, requiring supplemental oxygen.

5) Hospitalized, which does not require supplemental oxygen.

6) Not hospitalized, limitation of activities.

7) Not hospitalized, without limitations in activities.

Duration in days of. SARS-CoV-2 PCR positivity, hospitalization stay; mechanical ventilation supply; of oxygen use and Mortality rate.

Blood analysis parameters. Blood analysis is performed on hospitalization and discharging days.

The parameters of the study are filled every day on the individual patient cards -Questionnaire on symptomatology associated with COVID-19, at the visit of the patients.

\section{Statistical Analysis}

Data was processed by using patient data files. Parametric data was represented by mean values, normally distributed. Results were assessed by mean score for each day for each symptom for both groups for 21 days. Two tailed z-test was performed to determine whether the means of the two groups are different. $\mathrm{P}$-values less than 0.05 were considered significant with confidence interval of 95\%. All statistical analyses were performed using SPSS 22 software (SPSS, Chicago, IL, USA). 


\section{Results}

\subsection{Patients Characteristics}

Mean age of the recruited patients was $61.7 \pm 14.9$ year with range 40 to 89 years.

Mean age of Viusid-Asbrip group was $60 \pm 14$.1 years, while mean age of Control group was $64.9 \pm 16.2$ years $(\mathrm{p}=0.25)$. In both groups, the average post-infection day for starting therapy was approximately late 7 days. The disease severity measured in first day of hospitalization is equal for both group: $\mathrm{Vi}$ usid-Asbrip group, CSS $=8.38 \pm 1.88$, Control group, CSS $=8.50 \pm 1.57(\mathrm{p}=$ 0.79), see Table 1.

\subsection{Primary Outcome Measures}

Clinical Improvement as assessed by change in each symptom score: Investigated four symptom scores show much better results for Viusid-Asbrip group in all symptom diagrams, see Figure 1 . The difference is significant $(\mathrm{p}<0.05)$ between next days; Cough: day 7 - day 18; Dyspnea: day 11 - day 15; Fatigue: day 6, and day 10 - day 21; Fever: N.S., with best difference in days 4 - 6 and 13 -15 .

(a)

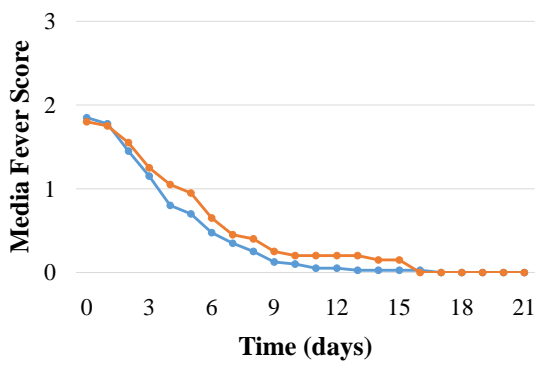

(c)

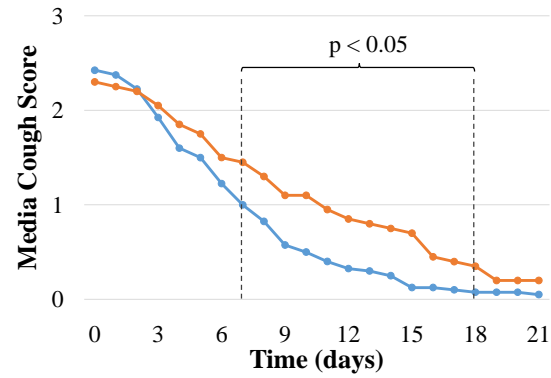

(b)

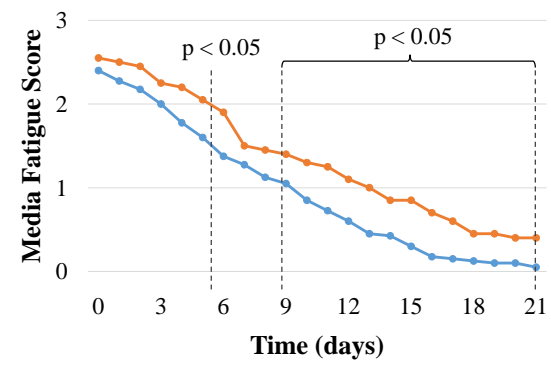

(d)

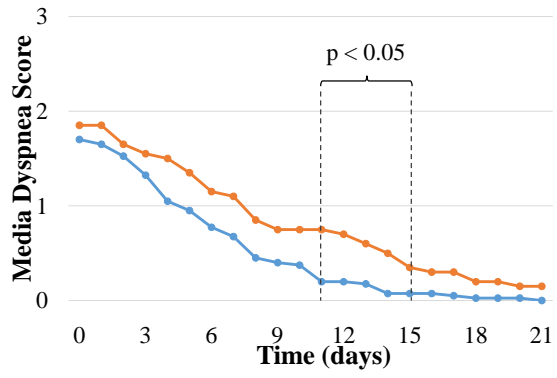

-Viusid + Asbrip $\_$Control

Figure 1. Evolution of COVID-19 Symptom Scores of Viusid-Asbrip group and Control group over 21 days of follow-up. (a) Evolution of fever Score (temperature in ${ }^{\circ} \mathrm{C}$ ) based on a scale $0-3: 0<36.7 ; 1=36.7-37.8 ; 2=37.8-38.9 ; 3>38.9$; (b) Fatigue Score on a 0 3 scale: $0=$ no fatigue, $1=$ mild fatigue, $2=$ moderate fatigue, $3=$ severe fatigue; (c) Cough Score on a scale 0 - 3: $0=$ no cough, $1=$ mild, $2=$ moderate, $3=$ severe; (d) Dyspnea Score based on a scale $0-3: 0=$ no dyspnea, $1=$ with moderate intensity exercise, $2=$ with walking on flat surface, 3 = with getting dressed or daily activities. P-values $<0.05$ were considered significant with confidence interval of $95 \%$. 
Table 1. Demographics characteristics of the patients in both groups.

\begin{tabular}{cccc}
\hline Outcome parameter & $\begin{array}{c}\text { Viusid-Asbrip } \\
n=40\end{array}$ & $\begin{array}{c}\text { Control } \\
n=20\end{array}$ & P value \\
\hline Mean age & $60 \pm 14.1$ & $64.9 \pm 16.2$ & NS \\
Men/ Woman & $15 / 25$ & $12 / 8$ & - \\
Disease severity - CSS & $8.38 \pm 1.88$ & $8.50 \pm 1.57$ & NS \\
Mild/ moderate disease & $3 / 37$ & $5 / 15$ & - \\
\hline
\end{tabular}

P-values $<0.05$ were considered significant with confidence interval of $95 \%$.

Symptom resolution time: Symptom resolution time (SRT) is defined by the number of days required to reach average value of each symptom score less than 0.25. In Table 2 SRT score shows much better results of Viusid-Asbrip group compare with Control group for all 4 symptoms.

Composite Symptom Score: The diagram of CSS showed the regression of the disease of both groups, Figure 2. The significantly lower results in favor of Viusid-Asbrip group are in whole diapason between day 4 and day $21(\mathrm{p}<0.05)$.

Time to semi-recovery. Time to semi-recovery is defined by the number of days required to reach a $50 \%$ reduction in the Composite Symptom Score. Table 2 shows better results of Viusid-Asbrip group vs Control group (6.07 \pm 2.77 vs. $8.35 \pm 2.94, \mathrm{p}=0.02$ ).

Time to recovery. Time to recovery is defined by the number of days required to reach Composite Symptom Score less than 1.00 value. Table 2 shows better results of Viusid-Asbrip group vs. Control group (14.05 \pm 4.15 vs. $19.25 \pm 2.12$, p $=0.0001)$.

\subsection{Secondary Outcome Measures}

Most often complication of both groups were pneumonia and respiratory failure (respiratory system); rhythm and conduction disorders, abnormalities in blood pressure (circulatory system); transient ischemic disorders, polyneuropathy (nervous system); strong increase of the liver enzyme ALAT which is due to the infectious disease and medications intake (blood analysis).

Hospitalization days. Average number of Hospitalization days of Viusid-Asbrip group are less than Control group $(9.05 \pm 2.58$ vs. $12.75 \pm 4.44, \mathrm{p}=0.0003$, Table 2).

Oxigen use days: Average number of Oxigen use days of Viusid-Asbrip group are less than Control group ( $4.73 \pm 1.56$ vs. $8.09 \pm 4.66, \mathrm{p}=0.02$, Table 2 ).

Blood analysis parameters. Blood analysis was done on the base / hospitalization day and on the discharging day. Because of different hospital stay is difficult to evaluate results. Nevertheless, both groups are improving their blood analysis parameters with non-significant difference. Most relevant parameters related with COVID-19 complications are represented in Figure 3: Fibrinogen (a), D-Dimer (b), Erythrocyte Sedimentation rate (ESR; c) and Total Bilirubin (d). 
Fibrinogen and D-dimer, which levels are significantly elevated in patients with deep venous thrombosis, pulmonary embolism, disseminated intravascular coagulation [31], complications which are seriously endanger the life of COVID-19 patients. The increment of ESR and TBIL is also associated with severe coronavirus disease 2019 [32] [33]. Diagrams of these four parameters show no significant differences for Viusid-Asbrip versus Control group. Focus on the normal range, better levels were observed of Viusid and Asbrip group on the discharging day in comparison with control group, that presented a tendency to increase the levels of these two parameters. D-Dimer and ESR show a diminution and normalization of ranges in Viusid-Asbrip group and an increment in control group.

Table 2. Parameters of the study outcomes of Viusid-Asbrip and Control groups.

\begin{tabular}{cccc}
\hline Outcome parameter & $\begin{array}{c}\text { Viusid-Asbrip } \\
\text { days }\end{array}$ & $\begin{array}{c}\text { Control } \\
\text { days }\end{array}$ & P value \\
1. Fever & 8 & 10 & - \\
2. Cough & 14 & 19 & - \\
3. Dispnea & 11 & 18 & - \\
4. Fatigue & 16 & 21 & 0.02 \\
Time to semirecovery & $6.07 \pm 2.77$ & $8.35 \pm 2.94$ & 0.0001 \\
Time to recovery & $14.05 \pm 4.15$ & $19.25 \pm 2.12$ & 0.0003 \\
Hospitalization days & $9.05 \pm 2.58$ & $12.75 \pm 4.44$ & 0.02 \\
\hline Oxigen use days & $4.73 \pm 1.56$ & $8.09 \pm 4.66$ & \\
\hline
\end{tabular}

P-values $<0.05$ were considered significant with confidence interval of $95 \%$.

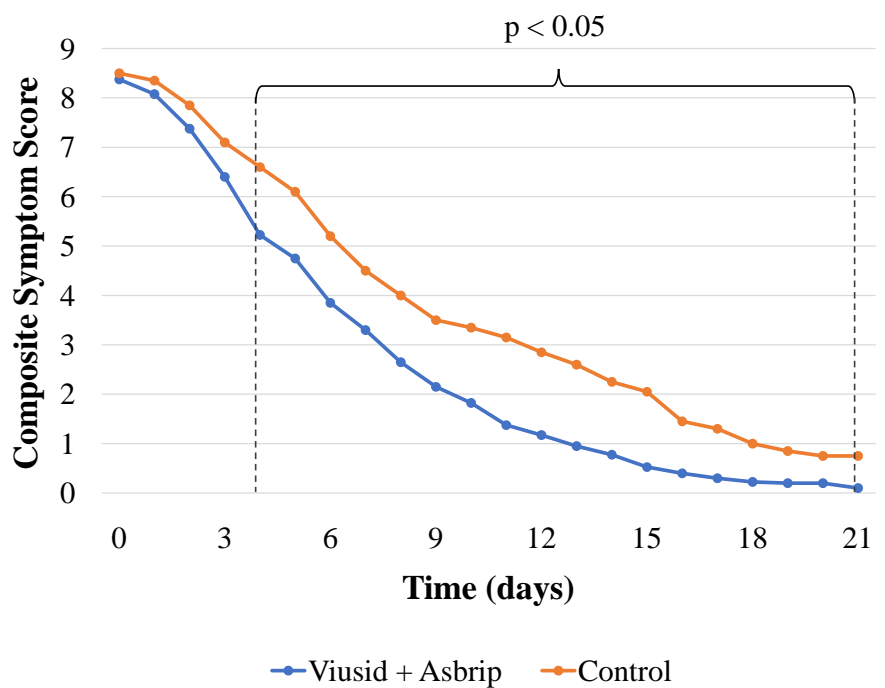

Figure 2. Evolution of Composite Symptom Score of Viusid-Asbrip and Control groups for 21 days follow-up. The sum of fever, fatigue, cough and dyspnea COVID-19 related symptom scores, assessed every day up to 21 days: ranges from 0 to 12 . $\mathrm{P}$-values $<0.05$ were considered significant with confidence interval of $95 \%$. 
(a)

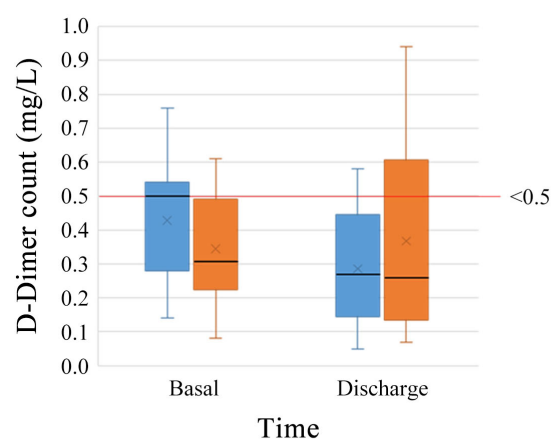

(c)

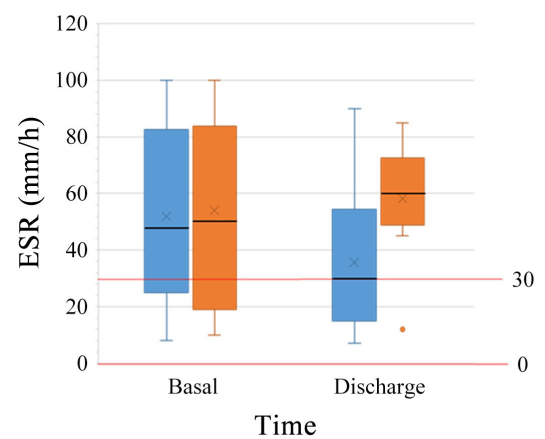

(b)

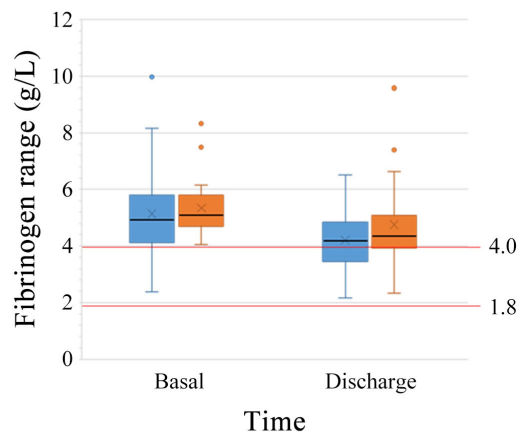

(d)

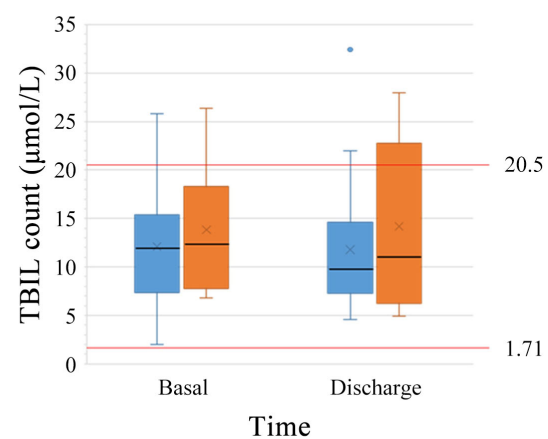

$=$ Viusid + Asbrip $\unrhd$ Control Group

Figure 3. Evolution of biomarkers related with the COVID-19 complications on hospitalization day and on discharging day in Viusid-Asbrip group vs Control group. (a) Fibrinogen levels measured at basal hospitalization time and at discharge time for Viusid-Asbrip group versus Control group; (b) D-Dimer count measured at basal hospitalization time and at discharge time for Viusid-Asbrip group versus Control group; (c) Erythrocyte Sedimentation Rate levels measured at basal hospitalization time and at discharge time for Viusid-Asbrip group versus Control group; (d) Total Bilirubin count measured at basal hospitalization time and at discharge time for Viusid-Asbrip group versus Control group. The normal range of Fibrinogen, D-Dimer, ESR and TBIL is represented with red lines ( 2.0 to $4.0 \mathrm{~g} / \mathrm{L} ; 0.0$ to $0.5 \mathrm{mg} / \mathrm{L} ; 0$ to $30 \mathrm{~mm} / \mathrm{h} ; 1.71$ to 20.5 respectively). The ranges of normal values may vary slightly between different laboratories.

\section{Safety}

Some published trials showed that Viusid was well tolerated, and only minor transient adverse events such as nausea and diarrhea were reported [22] [23]. Other trial concluded that Viusid tolerability was excellent, and no severe adverse event was reported [34]. The present trial states some side effects, which are more exactly the symptoms of the disease, because they are available since the first day. The trial reported for some frequent side effects in both groups: palpitations, tachycardia, and dizziness, available for average 8 days; insomnia and nervousness, available for average 12 days. The results of the study showed that the Viusid and Asbrip intervention products were safe since they did not show significant alteration in biochemical laboratory parameters (hematic biometry, liver enzimes, renal function, metabolic parameters), or adverse effects, in 
trial patients undergoing treatment. In conclusion Viusid and Asbrip are safe products, well tolerated and do not cause side effects.

\section{Discussion}

Modern advanced medicine has proved powerless against the new coronavirus SARS-CoV-2. Healthcare professionals felt an urgent need to find effective treatment for their patients. In some countries, there has been chaos in the selection of medicines and natural products for the treatment of patients with COVID-19, with various variants and combinations being tried, mostly with low effect. Of course, vaccines have high hopes for solving the problem of the coronacrisis. But at the moment we have millions of patients infected with the coronavirus who need to be cured.

In a randomized clinical trial, we evaluated the effect of the natural products Viusid and Asbrip in mild to moderate COVID-19 patients. The results of Viusid-Asbrip group compared to control group shows that it can help to favors the recovery of the COVID-19 patients and reduced the hospital stay. The adjuvance of Viusid and Asbrip showed better results compared with the standard clinical approach alone, most of them significantly: average Symptom Resolution time is shorter with 28\%; Time for Semi-recovery is shorter with 27.3\% ( $\mathrm{P}=0.02)$; Time for Recovery is shorter with $27 \%(\mathrm{P}=0.0001)$; Hospitalization days are less with $29 \%(P=0.0003)$; Days for oxigen use are less with $41.5 \%(P=0.02)$. No side effects were noted and mortality is missing. It should be noted that there is some difference in percent of decreasing the hospital stay of Viusid-Asbrip group vs control group in our trial, compare with Ecuador trial, $29 \%$ vs. 50\% [27]. This is due to the differences in the study design of both trials, concerning to the basic condition of the patients.

\section{Conclusion}

The products Viusid and Asbrip can be added to the standard treatment of COVID-19 disease and can contribute to faster recovery of the patients, decreasing of the hospital stay and milder course of the disease, with good safety and tolerability. Due to the small sample size, it is necessary to conduct other trials with more patients to confirm the results.

\section{Ethics Statement}

The study protocol was approved by the Ethics Commission of MBAL "Sveti Mina” (RCT001/P4/2020). Patient's personal information was entered as codes in the database, and the identities of patients were anonymous. The study was carried out under the principles of the Declaration of Helsinki.

\section{Data Availability}

Data are available on request to the corresponding author. 
This clinical trial is registered under identifier (NCT number):

https://ClinicalTrials.gov NCT04701502.

\section{Conflicts of Interest}

The authors declare no conflicts of interest regarding the publication of this paper.

\section{References}

[1] WHO (2020) Pneumonia of Unknown Cause-China. https://www.who.int/csr/don/05-january-2020-pneumonia-of-unkown-cause-china len

[2] WHO (2020) Naming the Coronavirus Disease (COVID-19) and the Virus That Causes It.

https://www.who.int/emergencies/diseases/novel-coronavirus-2019/technical-guida nce/naming-the-coronavirus-disease-(covid-2019)-and-the-virus-that-causes-it

[3] Gorbalenya, A.E., Baker, S.C., et al. (2020) The Species Severe Acute Respiratory Syndrome-Related Coronavirus: Classifying 2019-nCoV and Naming It SARS-CoV-2. ICTV. Nature Microbiology, 5, 536-544. https://doi.org/10.1038/s41564-020-0695-Z

[4] Liu, Y.-C. (2020) COVID-19: The First Documented Coronavirus Pandemic in History. Biomedical Journal, 43, 328-333.

https://www.sciencedirect.com/science/article/pii/S2319417020300445 https://doi.org/10.1016/j.bj.2020.04.007

[5] Guan, W., et al. (2020) Clinical Characteristics of Coronavirus Disease 2019 in China. New England Journal of Medicine, 382, 1708-1720.

https://doi.org/10.1056/NEJMoa2002032

[6] Zhang, H., Penninger, J.M., Li, Y., Zhong, N. and Slutsky, A.S. (2020) Angiotensin-Converting Enzyme 2 (ACE2) as a SARS-CoV-2 Receptor: Molecular Mechanisms and Potential Therapeutic Target. Intensive Care Medicine, 46, 586-590. https://doi.org/10.1007/s00134-020-05985-9

[7] Wan, Y., Shang, J., Graham, R., Baric, R.S. and Li, F. (2020) Receptor Recognition by Novel Coronavirus from Wuhan: An Analysis Based on Decade-Long Structural Studies of SARS. Journal of Virology, 94, e00127-20. https://doi.org/10.1128/JVI.00127-20

[8] Li, W., et al. (2003) Angiotensin-Converting Enzyme 2 Is a Functional Receptor for the SARS Coronavirus. Nature, 426, 450-454.

https://www.nature.com/articles/nature02145 https://doi.org/10.1038/nature02145

[9] Kuba, K. (2005) A Crucial Role of Angiotensin Converting Enzyme 2 (ACE2) in SARS Coronavirus-Induced Lung Injury. Nature Medicine, 11, 875-879.

https://www.nature.com/articles/nm1267 https://doi.org/10.1038/nm1267

[10] Zhou, P., et al. (2020) A Pneumonia Outbreak Associated with a New Coronavirus of Probable Bat Origin. Nature, 588, E6.

[11] Huang, C., et al. (2020) Clinical Features of Patients Infected with 2019 Novel Coronavirus in Wuhan, China. The Lancet, 395, 497-506. https://doi.org/10.1016/S0140-6736(20)30183-5

[12] NIH (2020) COVID-19 Treatment Guidelines. National Institutes of Health (NIH). 
https://covid19treatmentguidelines.nih.gov/introduction

[13] Yan, J.-M., et al. (2020) Discovery of Anti-2019-nCoV Agents from 38 Chinese Patent Drugs toward Respiratory Diseases via Docking Screening. Preprints, 2020, Article ID: 2020020254. https://www.preprints.org/manuscript/202002.0254/v2 https://doi.org/10.20944/preprints202002.0254.v2

[14] Chen, H. and Du, Q. (2020) Potential Natural Compounds for Preventing 2019-nCoV Infection. Preprints, 2020, Article ID: 2020010358.

https://www.preprints.org/manuscript/202001.0358/v1 https://doi.org/10.20944/preprints202001.0358.v3

[15] Otsuki, K. and Iahida, N. (1988) Inhibitory Effect of Glycyrrhizin on Polypeptide Phosphorylation by Polypeptide-Dependent Protein Kinase (Kinase P) in Vitro. Biochemical and Biophysical Research Communications, 157, 597-604.

https://pubmed.ncbi.nlm.nih.gov/2849432 https://doi.org/10.1016/S0006-291X(88)80292-4

[16] Cinatl, J., Morgenstern, B., Bauer, G., Chandra, P., Rabenau, H. and Doerr, H.W. (2003) Glycyrrhizin, an Active Component of Liquorice Roots, and Replication of SARS-Associated Coronavirus. The Lancet, 361, 2045-2046.

https://pubmed.ncbi.nlm.nih.gov/12814717 https://doi.org/10.1016/S0140-6736(03)13615-X

[17] Pagano, M.A., Tibaldi, E., Palù, G. and Brunati, A.M. (2013) Viral Proteins and Src Family Kinases: Mechanisms of Pathogenicity from a "Liaison Dangereuse". World Journal of Virology, 2, 71-78. https://pubmed.ncbi.nlm.nih.gov/24175231 https://doi.org/10.5501/wjv.v2.i2.71

[18] Li, X., Yang, M., Yu, Z., Tang, S., Wang, L., Cao, X. and Chen, T. (2017) The Tyrosine Kinase Src Promotes Phosphorylation of the Kinase TBK1 to Facilitate Type I Interferon Production after Viral Infection. Science Signaling, 10, eaae0435. https://pubmed.ncbi.nlm.nih.gov/28049762/ https://doi.org/10.1126/scisignal.aae0435

[19] Sviridov, D., Y.I. Miller, Ballout, R.A., Remaley, A.T. and Bukrinsky, M. (2020) Targeting Lipid Rafts-A Potential Therapy for COVID-19. Frontiers in Immunology, 11, Article ID: 574508. https://pubmed.ncbi.nlm.nih.gov/33133090/ https://doi.org/10.3389/fimmu.2020.574508

[20] Liu, Z., et al. (2014) Effects of Glycyrrhizin Acid and Licorice Flavonoids on LPS-Induced Cytokines Expression in Macrophage. China Journal of Chinese Materia Medica, 39, 3841-3845. https://pubmed.ncbi.nlm.nih.gov/25612451

[21] Richard, S.A. (2021) Exploring the Pivotal Immunomodulatory and Anti-Inflammatory Potentials of Glycyrrhizic and Glycyrrhetinic Acids. Mediators of Inflammation, 2021, Article ID: 6699560. https://doi.org/10.1155/2021/6699560

[22] Gomez, V., et al. (2010) Antioxidant and Imnunodulatory Effects of Viusid in Patients with Chronic Hepatitis C. World Journal of Gastroenterology, 16, 2638-2647. https://www.researchgate.net/publication/44644735 Antioxidant and immunomo dulatory effects of Viusid in patients with chronic hepatitis C https://doi.org/10.3748/wjg.v16.i21.2638

[23] Gomez, E., et al. (2011) Viusid, a Nutritional Supplement, Increases Survival and Reduces Disease Progression in HCV-Related Decompensated Cirrhosis: A Randomised and Controlled Trial. BMJ Open, 1, E000140.

https://www.ncbi.nlm.nih.gov/pmc/articles/PMC3191588

https://doi.org/10.1136/bmjopen-2011-000140

[24] Ocampo, L., et al. (2017) Effects of Glycyrrhizic Acid (Viusid-Vet ${ }^{\circledast}$ Powder) on the 
Reduction of Influenza Virus Spread and on Production Parameters in Pigs. Veterinaria México $O A$, 4. https://doi.org/10.21753/vmoa.4.1.373

[25] Gomez, J., et al. (2012) Effectiveness of Glycyrrhizinic Acid (Glizigen) and an Immunostimulant (Viusid) to Treat Anogenital Warts. International Scholarly Research Notices, 2012, Article ID: 863692.

https://www.hindawi.com/journals/isrn/2012/863692

https://doi.org/10.5402/2012/863692

[26] Brunswick Laboratories, Certificate of Analysis, 08/08/2014. http://www.davidagroup.com/viusid-vet/assets/images/catalogos/ORAC-VIUSID.p df

[27] Kobakova, A., et al. (2020) The Role of Immunostimulants in the Complex Therapy of Covid 19-A Clinical Case. Pharmacia, 67, 233-237.

https://pharmacia.pensoft.net/article/58024 https://doi.org/10.3897/pharmacia.67.e58024

[28] Crespo, A., et al. (2020) New Alternative for Treating COVID-19 in Ecuador. InterAmerican Journal of Medicine and Health, 3, e202003013.

https://www.iajmh.com/iajmh/article/view/82

[29] Morente, J. and Monje, E. (2005) Clinical Study to Assess the Efficacy and Tolerance of the Products Asbrip and Viusid in the Treatment of Chronic Pharingites. February to June. DOCPLAYER.

https://docplayer.net/162899692-Clinical-study-to-assess-the-efficacy-and-tolerance -of-the-products-asbrip-and-viusid-in-the-treatment-of-chronic-pharyngitis-februa ry-to-june-2005.html

[30] Safety and Efficacy of Viusid and Asbrip in Hospitalized Patients Infected by SARS-Cov-2 with COVID-19. ClinicalTrials.gov, NCT04407182.

https://clinicaltrials.gov/ct2/show/NCT04407182?cond=viusid+asbrip\&draw=2\&ra $\underline{\mathrm{n}=2}$

[31] Moresco, R., et al. (2003) D-Dimer and Its Relationship to Fibrinogen/Fibrin Degradation Products (FDPs) in Disorders Associated with Activation of Coagulation or Fibrinolytic Systems. Journal of Clinical Laboratory Analysis, 17, 77-79.

https://pubmed.ncbi.nlm.nih.gov/12696076 https://doi.org/10.1002/jcla.10072

[32] Lapić, I., Rogić, D. and Plebani, M. (2020) Erythrocyte Sedimentation Rate Is Associated with Severe Coronavirus Disease 2019 (COVID-19): A Pooled Analysis. Clinical Chemistry and Laboratory Medicine, 58, 1146-1148.

https://pubmed.ncbi.nlm.nih.gov/32386190/ https://doi.org/10.1515/cclm-2020-0620

[33] Paliogiannis, P. and Zinellu, A. (2020) Bilirubin Levels in Patients with Mild and Severe Covid-19: A Pooled Analysis. Liver International, 40, 1787-1788.

https://pubmed.ncbi.nlm.nih.gov/32304343/

https://doi.org/10.1111/liv.14477

[34] Gomez, E. (2008) Viusid, a Nutritional Supplement, in Patients with Chronic Hepatitis C and Cirrhosis. A Randomized and Controlled Study. Journal of Hepatology, 48, S124. https://doi.org/10.1016/S0168-8278(08)60316-5 https://www.cochranelibrary.com/central/doi/10.1002/central/CN-00653149/full?co okiesEnabled 